E N C R U C I J A D A

REVISTA ELECTRÓNICA DEL

CENTRO DE ESTUDIOS EN

ADMINISTRACIÓN PÚBLICA $28^{\circ}$ NÚMERO Enero-Abril 2018

Revista Electrónica del Centro de Estudios en Administración Pública de la Facultad de Ciencias Políticas y Sociales, Universidad Nacional Autónoma de México

\title{
El gobierno electrónico municipal en Toluca, Estado de México, desde la perspectiva ciudadana
}

Leticia Contreras Orozco*

Recibido: 12 de septiembre de 2017 Aceptado: 12 de diciembre de 2017

\section{Resumen}

El objetivo central de este trabajo es conocer la percepción ciudadana sobre el funcionamiento del portal electrónico del gobierno municipal de Toluca, a fin de identificar si los ciudadanos hacen uso del portal y cómo lo utilizan; se enfatizó en tres aspectos: información, prestación de servicios y participación. Para alcanzar el objetivo planteado, se determinó realizar un estudio exploratorio de carácter cuantitativo, lo cual permitió obtener entre otros resultados, que desde la percepción ciudadana el portal gubernamental es más utilizado para simplificar algunos trámites, seguido de proporcionar información y escasamente propicia la participación de los ciudadanos.

\section{Palabras clave}

Gobierno electrónico, gobierno local, Tecnologías de la Información y Comunicación, participación ciudadana, innovación.

\section{Abstract}

The main objective of this work is to know the public perception about the functioning of the electronic portal of the municipal government of Toluca in order to identify if the citizens make use of the portal and how they use

\footnotetext{
* Profesora-Investigadora de tiempo completo en Administración Pública y Gestión Pública, en la Universidad Autónoma del Estado de México. Líder del Cuerpo Académico: Estado, gestión y TIC en las sociedades del siglo XXI.
} 
it. It was emphasized in three aspects: information, provision of services and participation. In order to reach the proposed objective, it was determined to carry out an exploratory study of a quantitative nature, which allowed to obtain, among other results, that from the citizen perception the governmental portal is more used to simplify some procedures, followed by providing information and scarcely conducive the participation of the citizens.

\section{Keywords}

E-government, local government, Information and Communication Technologies, citizen participation, innovation.

\section{Introducción}

En los años recientes, el uso de herramientas tecnológicas se ha hecho casi común. A partir del uso de internet y de la web 2.0 el intercambio de información puede realizarse de forma continua. Los progresos tecnológicos han generado que los gobiernos sean innovadores, que se modernicen, pues también son más visibles en un espacio virtual.

En este contexto, se considera importante estudiar al gobierno municipal de la capital de uno de los estados más poblados de México por la relevante cercanía de este ámbito de gobierno con los ciudadanos. Se parte del supuesto que la ciudadanía cada vez está más familiarizada con el uso de herramientas tecnológicas y por ello la simplificación de trámites o tener acceso a algún servicio mediante la web es cada día más común, sobre todo en un municipio urbano de las características de Toluca, capital del Estado, sin embargo, no necesariamente es así, por ello la pregunta de investigación que orientó este trabajo fue: ¿para qué usan los ciudadanos el portal electrónico y qué opinan de él?

La percepción de la ciudadanía es un aspecto poco abordado en los estudios sobre gobierno electrónico en el marco de la modernidad tecnológica en el siglo XXI. Se llevan a cabo evaluaciones técnicas de los portales web, existen metodologías diseñadas para llevar a cabo tales evaluaciones, se conoce el contenido y la posibilidad de interacción y transacción de los portales, sin embargo, el acercamiento con los ciudadanos para saber cómo perciben el uso de las herramientas tecnológicas y cómo las utilizan es una veta importante. Por ello, este trabajo tiene como objetivo conocer la percepción ciudadana sobre el funcionamiento del portal electrónico del gobierno municipal de Toluca, a fin de 
identificar si los ciudadanos hacen uso del portal y cómo lo utilizan, se enfatizó en tres aspectos: información, prestación de servicios y participación.

En la sección 2 del trabajo se describe la metodología seguida para la obtención de los resultados. En la sección 3 se aborda el tema de las Tecnologías de la Información y Comunicación (TIC), vinculadas con la innovación en la gestión local para resaltar los cambios que pueden generar en los gobiernos y las posibles ventajas de su utilización.

En la sección 4 se desarrolla el marco conceptual del gobierno electrónico, no es exhaustivo, pero permite destacar los elementos necesarios para el estudio. En el apartado 5 se desarrolla el estudio sobre el municipio de Toluca, se describen los resultados obtenidos y se presentan algunas tablas y gráficas de los aspectos que se consideran más importantes.

Finalmente, en el último apartado se presenta la interpretación de resultados con relación a la pregunta de investigación y los principales hallazgos, así como las conclusiones.

\section{Metodología}

Para alcanzar el objetivo planteado se determinó realizar un estudio exploratorio de carácter cuantitativo, a fin de indagar acerca de la opinión de los ciudadanos sobre el portal electrónico del gobierno municipal de Toluca, ya que dado el avance de la tecnología, se parte del supuesto que la ciudadanía cada vez está más familiarizada con el uso de herramientas tecnológicas y por ello la simplificación de trámites o tener acceso a algún servicio mediante la web es cada día más común; sin embargo, este aspecto es poco conocido cuando se llevan a cabo evaluaciones técnicas de los portales web.

La percepción ciudadana es entendida como la manifestación del sentir o de la opinión y actitud de los ciudadanos respecto a algún suceso o hecho que puede involucrarlos. Por ello se considera indispensable conocer qué piensan los ciudadanos del portal web que el gobierno municipal pone a su disposición, y cómo o para qué lo utilizan, ya que 
técnicamente puede significar que el gobierno está a la vanguardia, que es un gobierno moderno, pero el fin último debe ser la ciudadanía y su bienestar.

Para el desarrollo de esta investigación, se consideró como población total el número de ciudadanos del municipio de Toluca registrados en el padrón electoral al 31 de agosto de 2016, con la finalidad de que la encuesta se aplicara a mayores de edad; así, la población total fue de 595,924 ciudadanos.

Se realizó un muestreo probabilístico proporcional al tamaño de la población, con un nivel de confianza del $95 \%$ y un margen de error de $+/-4$. Derivado de ello, se aplicaron 600 encuestas cara a cara a los ciudadanos que conocieran el portal electrónico del gobierno municipal, con el objetivo de conocer si los ciudadanos están familiarizados con el uso de la página electrónica, si han hecho uso de ella y conocer su opinión acerca de su funcionamiento.

Las preguntas de la encuesta se agruparon en cuatro rubros, las que permitieron tener un conocimiento general sobre los ciudadanos muestra, y las preguntas que enfatizaron en tres aspectos: información, prestación de servicios y participación que son los elementos de la dimensión externa del gobierno electrónico, la parte más visible.

La información define el perfil de la organización (Contreras, 2014), en este rubro se considera la información acerca de trámites, pagos o se puede tener acceso a algunos documentos. La prestación de servicios se refiere a un ámbito de gran diversidad, ya que cada gobierno pone al alcance de los ciudadanos los servicios que considera necesarios o aquellos en los que está en condiciones técnicas o normativas de proporcionar vía internet, de esa manera los ciudadanos pueden realizar pagos, trámites varios, o bien emitir quejas o sugerencias. La participación representa la oportunidad de crear canales permanentes de comunicación, un espacio en el que los ciudadanos pueden opinar y ser partícipes de la toma de decisiones. Por lo anterior, estos aspectos que son parte de las evaluaciones técnicas de los portales web, en este caso se priorizan desde la perspectiva del ciudadano. 


\section{Las Tecnologías de la Información y Comunicación y la innovación de la gestión pública local}

En los años recientes, las Tecnologías de la Información y Comunicación (TIC) se han vuelto un tema esencial vinculado a los cambios o a los procesos de innovación en los gobiernos, especialmente en aquellos que se consideran los más cercanos a los ciudadanos: los gobiernos locales.

Las nuevas tecnologías han transformado la forma de llevar a cabo la gestión pública, han cobrado importancia porque se han constituido en un recurso indispensable para sistematizar procedimientos, simplificar trámites, reducir tiempos de espera y costos, permiten aumentar la eficiencia, eficacia y productividad, la calidad de los servicios públicos, la participación ciudadana o la transparencia y rendición de cuentas (Criado y Gil-García, 2013; Pando y Fernández, 2013).

Para Villoria y Ramírez (2013), la utilización de las TIC se ha convertido en una característica de los gobiernos y las administraciones públicas de este siglo; mediante su uso se han diversificado las formas de gestión y se han constituido nuevos canales para que la ciudadanía pueda expresarse, además de tener la posibilidad de una comunicación directa con sus gobernantes.

El uso de Internet y las nuevas tecnologías han modificado la relación ciudadanogobierno, ya que antes de la aparición de estas herramientas, era muy complicado que existiera una comunicación directa, "Internet y la web [world wide web] hacen posible que las dependencias y organismos gubernamentales reestructuren su interacción con los ciudadanos" (Fountain, 2013:105).

Actualmente, las TIC son herramientas de uso cotidiano, constituyen la base técnica que permiten a los gobiernos construir nuevos modelos de gestión, innovando en la prestación de servicios y el acceso a la información.

Mulgan y Albury (cit. por Ramírez, 2010:9), consideran que los procesos de innovación en el sector público obedecen a razones como: 
- Responder más eficazmente a los cambios de las necesidades públicas y las crecientes expectativas ciudadanas;

- Contener los costes y aumentar la eficiencia;

- Mejorar la prestación y los resultados de los servicios públicos;

- Aprovechar todo el potencial de las TIC.

Por ello, a partir del uso de las TIC, se generan procesos de innovación institucional, de innovación en la gestión y de transformación de las organizaciones públicas; sin embargo, es indispensable considerar que estos procesos son posibles si se vinculan a objetivos claros, la incorporación de tecnología por sí misma no es sinónimo de buenos resultados. Las nuevas tecnologías no sólo deben ser vistas como herramientas para la solución de problemas, sino también deben asumir un rol estratégico.

Las organizaciones públicas no son meros espacios en el vacío en los que se aplican tecnologías, que desencadenan determinados resultados. Las tecnologías, a su vez, se ven modificadas como consecuencia de su interacción con las organizaciones públicas y su entorno político, las personas que trabajan en ellas, así como los arreglos institucionales que se encuentran presentes dentro de una determinada arena de acción gubernamental (Criado y Gil-García, 2013:10)

Con la incorporación de las TIC en los gobiernos locales, se han generado diversos cambios, con el surgimiento específicamente de la Web 2.0, se abren otras oportunidades para el usuario como la posibilidad de interactuar a través de redes, blogs o wikis entre otras opciones, lo cual representa una oportunidad para generar mecanismos de participación directa a diferencia de otras esferas de gobierno más complejas (Mossberger, Wu y Crawford, 2013). Adicionalmente, el poder difusor de las TIC hace que los líderes locales adquieran cada vez mayor relevancia (Pando y Fernández, 2013), sean más visibles y las decisiones se conozcan y se difundan rápidamente.

Aspectos como el liderazgo, la gestión del capital humano, el diseño y cambio organizativo, las relaciones interadministrativas e intergubernamentales, la comunicación y el marketing de servicios, así como la transparencia no se pueden ni se deben entender de la misma manera en un entorno en el que las 
administraciones públicas son usuarias intensivas de tecnologías de información (Criado y Gil-García, 2013:43).

Los cambios en la gestión local a través de la incorporación de las TIC, redefinen los instrumentos y la forma en que se prestan los servicios; sin embargo, son sólo un medio al cual no debe atribuirse una función casi mágica (Pando, 2010), pero bajo una visión clara, con objetivos definidos y con una estrategia de gestión, puede dar paso a una nueva forma de atender las necesidades sociales: el gobierno electrónico.

\section{Marco conceptual del gobierno electrónico}

La idea de gobierno electrónico ha sido adoptada gradualmente con el objetivo de hacer más eficientes los procesos administrativos, mejorar la calidad de los servicios y al mismo tiempo la calidad de vida de los ciudadanos. A través del gobierno electrónico se pretende crear una comunicación estrecha entre los ciudadanos y los funcionarios, lo cual permite "eliminar" estructuras jerárquicas y de esta forma ganar la confianza del ciudadano.

Aunque la idea más cercana al gobierno electrónico es el uso de la tecnología, no lo es todo. El gobierno electrónico implica un cambio en el paradigma de la gestión gubernamental (Naser y Concha, 2011); es una nueva forma de gobierno que involucra factores tecnológicos, organizativos, institucionales, humanos y contextuales (Criado y Gil-García, 2013).

Encontrar una definición única de gobierno electrónico no es fácil, ya que no se refiere únicamente a la parte tecnológica, aunque este aspecto es fundamental tal y como lo subrayan por ejemplo la Organización para la Cooperación y el Desarrollo Económico (OCDE) y el Banco Mundial, pero también son importantes los aspectos organizativos; para Gil-García (2012) el gobierno electrónico es un fenómeno socio- técnico.

El gobierno electrónico puede ser definido como "la aplicación de las TIC para lograr una administración efectiva, eficiente y transparente de todos los recursos del gobierno, así como también el acceso fácil y expedito a servicios públicos, para ciudadanos, empresas 
y otras entidades que interactúen con el gobierno" (Alfaro, Bustos, González y Loroño, 2005:14).

Sin embargo, para los fines de este trabajo se retoma el concepto de Ramió y Salvador, que consideran de manera específica el ámbito local, así, se entiende al gobierno electrónico como "la utilización de las TIC por parte de los gobiernos locales a través de la mejora de su gestión interna, de la oferta de servicios e información y de los sistemas de intercambio e interacción con los ciudadanos y con las organizaciones públicas y privadas" (2005:163).

El gobierno electrónico presenta dos dimensiones, la interior, que se vincula a la gestión interna de los gobiernos y la exterior en la que resaltan tres aspectos: la prestación de servicios, la oferta de información y la participación democrática (Contreras, 2014). La parte más visible de la gestión de los gobiernos locales a través de la implementación del gobierno electrónico se percibe a través de la dimensión exterior, a partir de la cual se pueden establecer relaciones de mayor proximidad con la ciudadanía, se puede mejorar la prestación de servicios y los ciudadanos tienen una opinión más certera sobre el actuar de su gobierno local.

El gobierno electrónico en el ámbito local transforma las relaciones internas, ya que permite que fluya la información entre unidades administrativas; también transforma las relaciones externas: la oportunidad de proporcionar mejores servicios. Asimismo, que los ciudadanos tengan acceso a información gubernamental, contribuye a la transparencia y la posibilidad de participar en los asuntos públicos genera mayor confianza en las acciones gubernamentales.

La gestión pública mediante la utilización de las TIC adquiere un matiz moderno e innovador, pero de manera más profunda bajo el modelo de gobierno electrónico que idealmente sería un instrumento que aporte a la construcción de gobiernos locales eficientes, transparentes, participativos y éticos al servicio de la ciudadanía.

La expresión más visible del gobierno electrónico son los portales web, de ahí la importancia de evaluarlos técnicamente y saber cómo funcionan; no obstante, una parte 
fundamental en la construcción y mejora de los portales debiera ser la opinión de los ciudadanos quienes son los principales beneficiarios de los servicios vía electrónica.

Hoy en día la mayoría de los gobiernos y organizaciones públicas cuentan con sitios web, algunos utilizados únicamente como medio de difusión, aunque lo ideal es que un portal gubernamental (con las ventajas que proporciona la web 2.0) sea dinámico e innovador, en donde el ciudadano pueda interactuar además de obtener información pública y tener al alcance servicios desde el lugar donde se encuentre.

En el gobierno electrónico, los portales web son probablemente el vínculo más importante entre el ciudadano y el gobierno, a través de ellos se da la interacción y el intercambio de información de manera directa, por ello se hace necesaria su constante actualización. Adicionalmente, uno de los aspectos más relevantes que puede impulsar el gobierno electrónico, es la participación de los ciudadanos en los asuntos públicos y en la toma de decisiones.

Los portales web deben adaptarse a las necesidades y expectativas de los ciudadanos, ya que ellos son los directamente beneficiados (Sandoval y Gil-García, 2009) y de quien se busca una participación más activa y directa. Derivado de lo anterior, es necesario señalar la importancia que tienen los mecanismos de evaluación de los portales gubernamentales, así como su avance y actualización. A través de una evaluación se identifican los aspectos que pueden ser mejorados.

La medición de los esfuerzos gubernamentales para incorporar la tecnología a su gestión, es necesaria para conocer sus avances o cómo se encuentran en comparación con otros gobiernos. Estrada (2007) considera que tal medición contribuye a la comprensión de la sociedad de la información, no obstante, las metodologías de medición pueden marcar diferentes enfoques.

Sandoval y Gil-García (2009), han desarrollado un modelo de evaluación de portales gubernamentales, entre los elementos que se consideran están: información, interacción, transacción, integración y participación política; la evaluación se desarrolla a partir del desglose de estos indicadores y la confrontación con el portal web y su contenido, esto ha permitido la elaboración de un ranking entre los gobiernos subnacionales en México para 
conocer los avances en esta materia. Es un orden de gobierno específico, pero también es un modelo que puede servir en otros contextos si se hacen adaptaciones.

Otra propuesta es la de Esteves (2005), quien se enfoca específicamente en gobiernos municipales y desarrolla una metodología que tiene por objetivo identificar el nivel de desarrollo de los servicios mediante el gobierno electrónico, esta propuesta identifica como fases la presencia, información urbana, interacción, e-democracia, cada una de ellas representa las capacidades de un gobierno municipal para proveer servicios.

La evaluación de portales web gubernamentales permitirá mejorar la calidad de los mismos, pero un aspecto que va más allá de la evaluación técnica es la opinión de los ciudadanos, objetivo de este trabajo, ya que es necesario conocer que tan vinculados están los ciudadanos con el uso de la tecnología y si esto representa la posibilidad de mayor cercanía con el gobierno local.

Scott (2006), ha señalado que los portales web de los gobiernos locales (en un estudio en Norteamérica) poseen un buen registro de información, pero los canales para la participación y aportación ciudadana son unidireccionales, por ello, el reto no sólo es la funcionalidad tecnológica, sino la posibilidad de que sean portales accesibles con información clara y precisa para el ciudadano y con posibilidades de interacción.

\section{Estudio en el Municipio de Toluca. Descripción de resultados}

El municipio de Toluca es uno de los 125 municipios en que se divide el Estado de México, es además su capital, tiene una extensión total de 420.14 kilómetros cuadrados que representan el $1.87 \%$ del total del territorio del Estado de México.

Toluca es el cuarto municipio más poblado del estado, tiene un total de 819,561 habitantes, siendo 394, 836 hombres y 424, 725 mujeres; de esa población, 94\% es urbana y $6 \%$ es rural. Asimismo, en el municipio de Toluca $2.9 \%$ de la población de más de cinco años de edad es hablante de alguna lengua indígena, este porcentaje equivale a un total de 18,816 personas, de las cuales 8,987 son hombres y 9,629 son mujeres; del total, 17,517 son bilingües al español, 210 son monolingües y 889 no especifican 
condición de bilingüismo. El porcentaje de la población alfabeta de 15 años y más en el Municipio de Toluca, ascendió a 96.01\% en el año 2010 (INEGI, 2010).

Para el estudio, con base en la muestra estadística calculada se aplicaron 600 cuestionarios durante los meses de enero y febrero del 2017 en el Municipio de Toluca, solamente a las personas mayores de edad que conocieran o que hubieran utilizado el Portal Web del gobierno municipal; fueron 311 mujeres que equivale al $45.70 \%$ de la población encuestada y 369 hombres, que equivale al $54.30 \%$ de los encuestados. Las preguntas permitieron tener un conocimiento general sobre los ciudadanos muestra y sobre cada uno de los aspectos de la dimensión exterior del gobierno electrónico: información, servicios y participación. Aquí se presentan los resultados de las más relevantes.

Uno de los aspectos contenidos en la encuesta fue el dato acerca de la edad, con la finalidad de saber si ésta puede ser un factor que influya en el uso del portal gubernamental. En la gráfica 1 se muestran los rangos de edades de las personas encuestadas, de ahí se deriva que las personas entre 18 y 39 años son las que han visitado o utilizado el Portal Web; en contraste con ello, se observa que las personas mayores de 40 años hacen menos uso del portal.

Gráfica 1. Edad de los encuestados

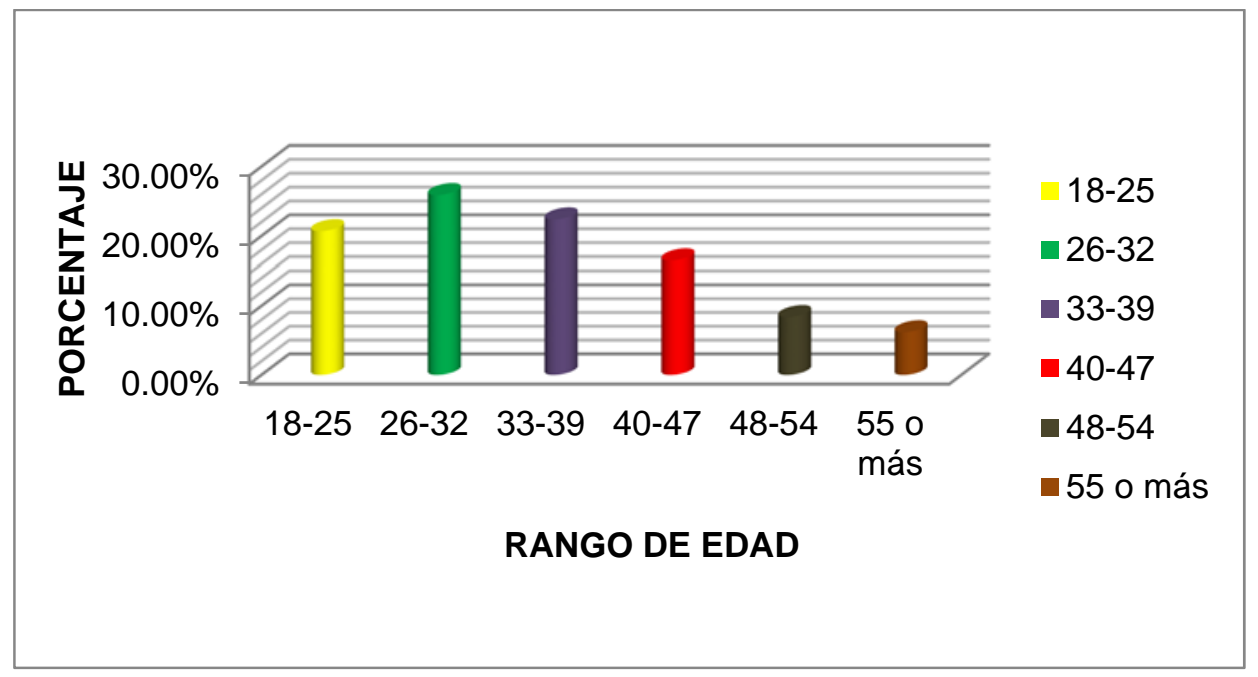

Fuente: Elaboración propia. 
Por otra parte, de la población encuestada el grado escolar comprende desde la educación básica hasta el posgrado. El mayor porcentaje de los usuarios del portal electrónico del gobierno municipal se concentra en personas con estudios de licenciatura, seguido por quienes poseen una carrera técnica y bachillerato respectivamente. El nivel básico es el de menor frecuencia como se aprecia en la gráfica 2. Derivado de lo anterior, se resalta que a mayor grado de estudios mayor es la incidencia de los ciudadanos que visitan la página electrónica del Municipio.

Gráfica 2. Escolaridad

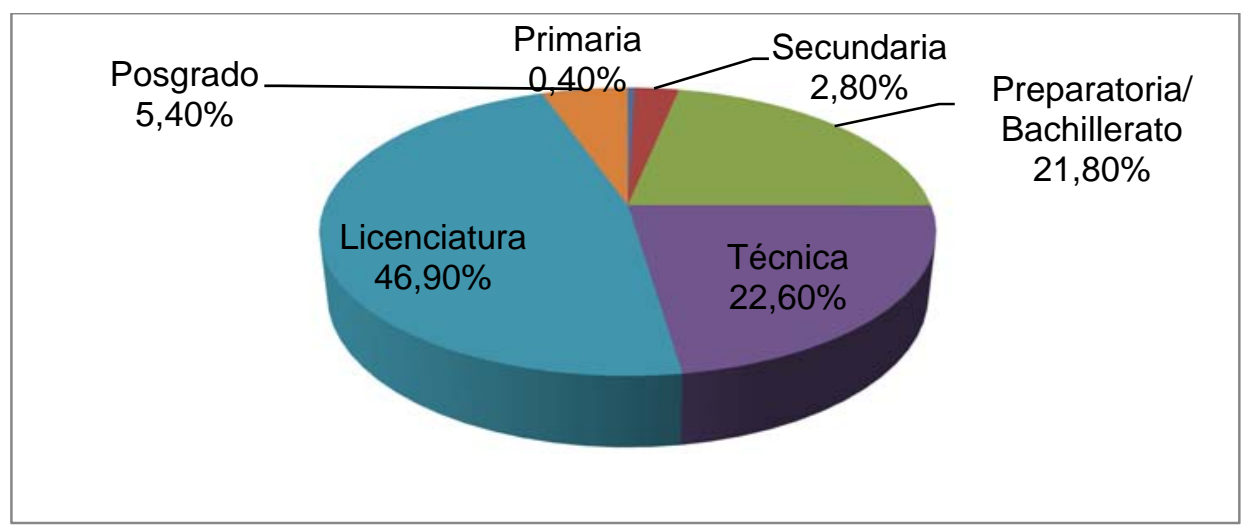

Fuente: Elaboración propia.

La ocupación de los ciudadanos encuestados es diversa, estos datos se concentran en la tabla 1, donde el mayor porcentaje se concentra en los empleados (de diferentes sectores) y servidores públicos. Las personas que ocupan un menor porcentaje de la muestra son aquellas cuya ocupación es el campo, trabajos no formales, algún oficio o son obreros. 
Tabla 1. Ocupación

\begin{tabular}{cc}
\hline OCUPACIÓN & PORCENTAJE \\
\hline Servidor público & $20.10 \%$ \\
Empleado & $22.60 \%$ \\
Obrero & $2.20 \%$ \\
Comerciante & $6.80 \%$ \\
Profesionista & $14.30 \%$ \\
Estudiante & $14.40 \%$ \\
Hogar & $9.10 \%$ \\
Desempleado & $2.90 \%$ \\
Jubilado o pensionado & $3.70 \%$ \\
Oficios & $2.20 \%$ \\
Campesino & $0.10 \%$ \\
Otro & $1.50 \%$ \\
Total & $100.0 \%$ \\
\hline
\end{tabular}

Fuente: Elaboración propia.

Para la aplicación de la encuesta fue indispensable que las personas utilizaran Internet y que conocieran o hubieran utilizado el Portal Web. La tabla 2 muestra los lugares en donde la población muestra utiliza Internet.

Tabla 2. Lugar donde se utiliza Internet

\begin{tabular}{cl}
\hline LUGAR & PORCENTAJE \\
\hline Casa & $37.90 \%$ \\
Trabajo & $27.30 \%$ \\
Establecimiento que ofrece el servicio & $3.40 \%$ \\
Wifi público & $4.70 \%$ \\
Móvil & $26 \%$ \\
Otro & $0.70 \%$ \\
Total & $100.0 \%$ \\
\hline
\end{tabular}

Fuente: Elaboración propia. 
La tabla anterior permite observar que la mayoría de las personas encuestadas cuentan con Internet en casa, que pueden hacer uso de éste también en el trabajo y que a través de su dispositivo móvil tienen acceso a Internet.

Respecto al conocimiento o uso del portal, únicamente el $60.6 \%$ la ha utilizado alguna vez, el $37.9 \%$ la utiliza de manera ocasional y únicamente el 1.5\% no la ha utilizado, sólo la conoce. La tabla 3 muestra qué actividades han realizado los encuestados.

Tabla 3. Actividades realizadas en la página de Internet

\begin{tabular}{cc}
\hline ACTIVIDADES & PORCENTAJE \\
\hline Conocer información sobre actividades del \\
gobierno municipal & $27 \%$ \\
Conocer información general del municipio & $17 \%$ \\
Pagar servicios & $13.4 \%$ \\
Realizar trámites & $29.3 \%$ \\
Participación/ interacción & $5 \%$ \\
Todas las anteriores & $8.3 \%$ \\
TOTAL & $100.0 \%$ \\
\hline
\end{tabular}

Fuente: Elaboración propia.

Como se puede observar, el Portal Web es más utilizado para realizar trámites y conocer información sobre las actividades del gobierno municipal siendo el pago de servicios y la interacción las actividades menos realizadas. Se resalta que menos del 10\% de la muestra ha realizado todas las actividades del listado.

Para conocer la opinión de los ciudadanos, se les preguntó qué calificación otorgarían al portal considerando la información que éste proporciona, la calificación más alta fue de 8 en una escala del 1 al 10, los resultados se muestran en la gráfica 3. 
Gráfica 3. Calificación de la página web respecto de la información que ofrece

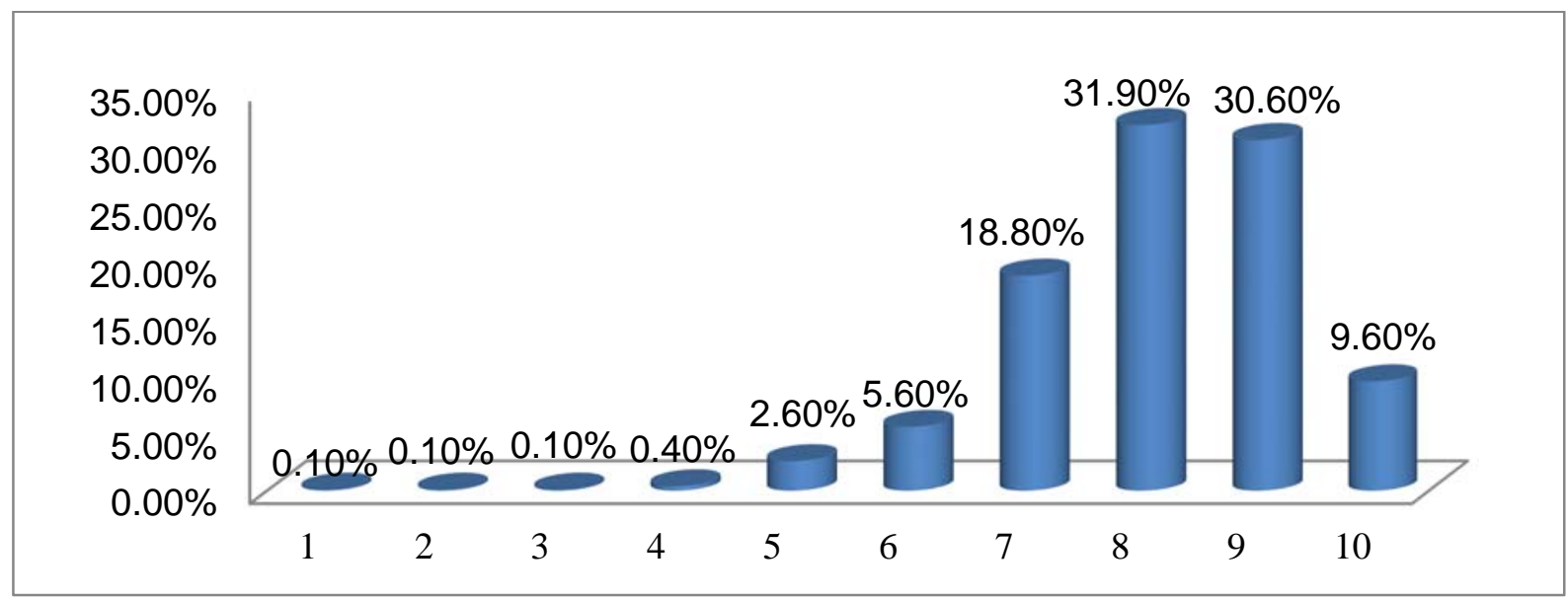

Fuente: Elaboración propia.

Asimismo, a quienes habían realizado algún trámite se les pidió señalar cómo consideraban el tiempo de respuesta obtenida al momento de realizar sus trámites, lo cual se aprecia en la gráfica 4 , donde el mayor porcentaje señala una respuesta de rápida a normal. Adicionalmente, los ciudadanos también otorgaron una calificación al portal considerando su experiencia en el trámite realizado, lo cual se refleja en la gráfica 5.

Gráfica 4. Tiempo de respuesta a trámite realizado

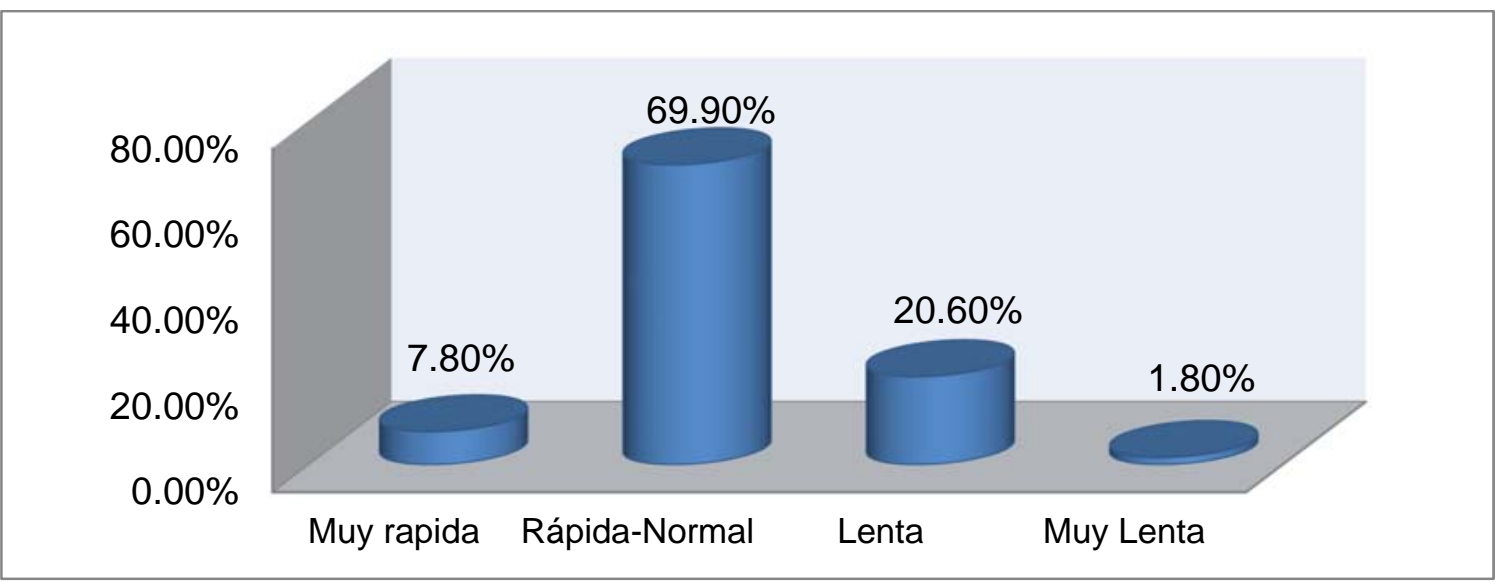

Fuente: Elaboración propia. 
Gráfica 5. Calificación asignada a página de Internet con base en el trámite realizado

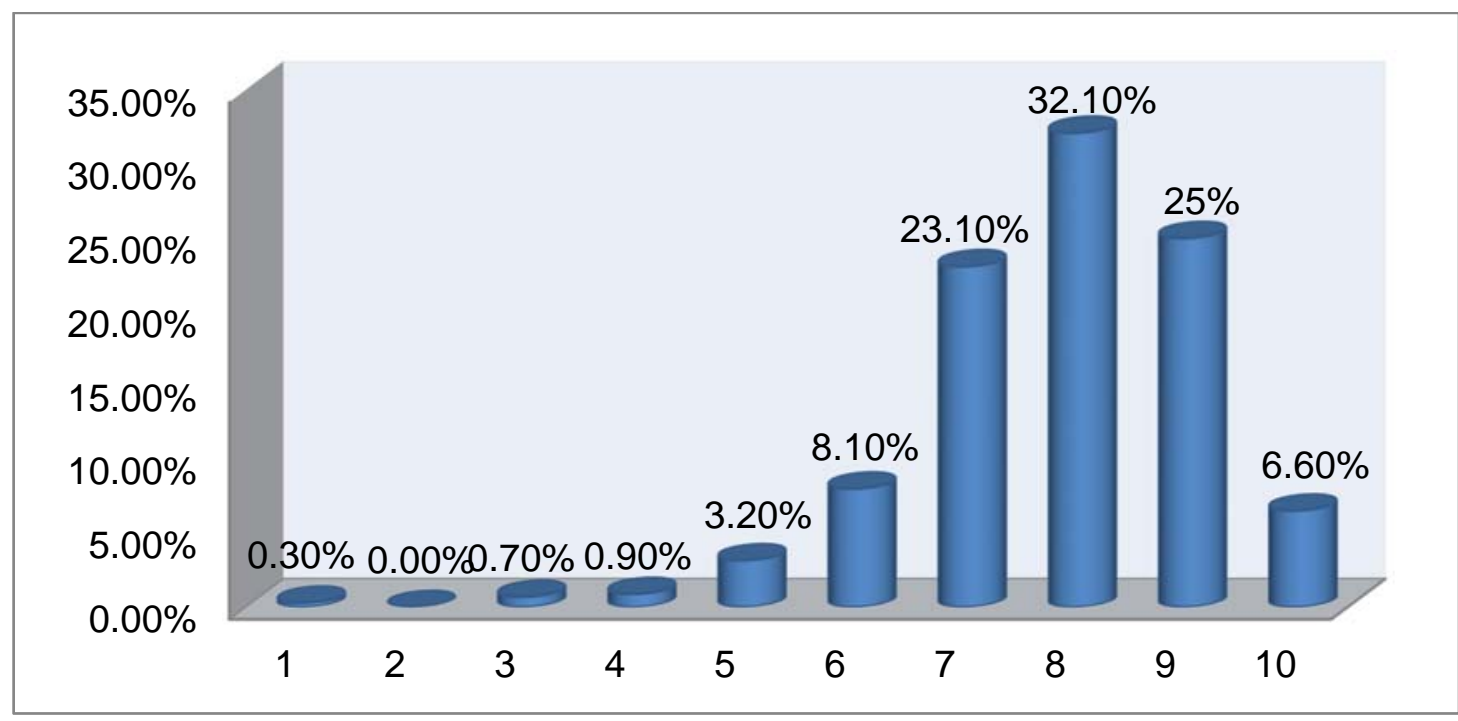

Fuente: Elaboración propia.

El tercer elemento en la dimensión externa del gobierno electrónico es la participación, para que esta pueda darse es necesario contar con espacios o canales de interacción entre el gobierno y los ciudadanos, por ello se preguntó a los encuestados acerca de la existencia de un espacio que propiciara dicha interacción. El 22\% respondió que si existen canales para interactuar, el $40 \%$ respondió que no existen y el $38 \%$ no sabe. Del grupo de personas que respondió afirmativamente sobre la posibilidad de interacción, sólo el 75\% pudo señalar claramente cuáles son los medios que permiten la interacción con el gobierno o con servidores públicos, los resultados se muestran en la tabla 4.

Tabla 4. Medios de interacción con ciudadanos

\begin{tabular}{c|c}
\hline Medios utilizados & Porcentaje \\
\hline Redes sociales & $45.8 \%$ \\
\hline Chat & $25.8 \%$ \\
\hline Correo electrónico & $10.8 \%$ \\
\hline Foros & $4.2 \%$ \\
\hline Otras & $13.4 \%$ \\
\hline
\end{tabular}

Fuente: Elaboración propia. 
Los resultados muestran que los ciudadanos consideran a las redes sociales como el medio principal de interacción con funcionarios. Con base en los datos anteriores, se solicitó emitir una calificación al portal gubernamental considerando las posibilidades de participación que tienen a través de ese medio, los resultados se presentan en la gráfica 6, en donde el más alto porcentaje señala una calificación de 7 en una escala de 1 a 10 donde 10 es la mejor calificación que se puede otorgar.

Gráfica 6. Calificación al portal web respecto a la participación ciudadana

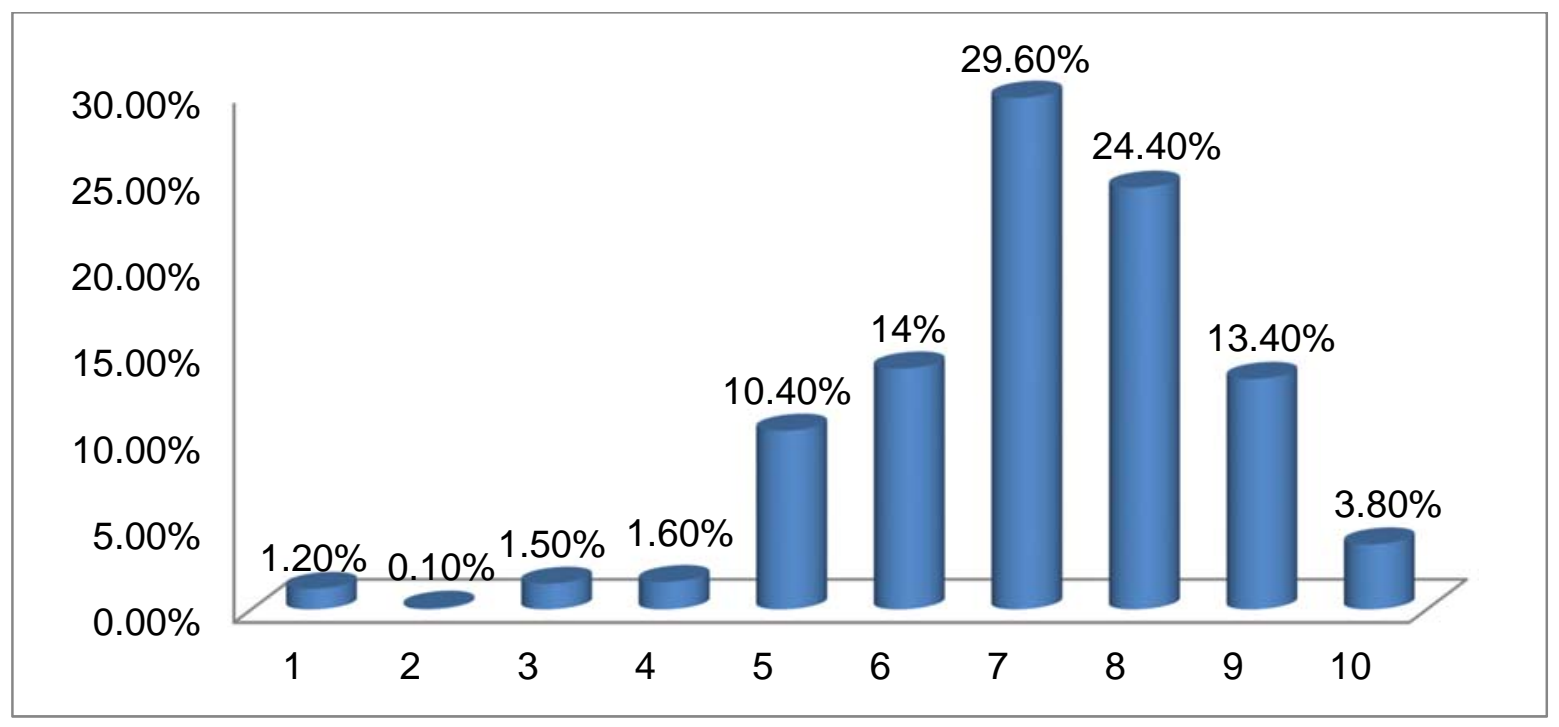

Fuente: Elaboración propia.

Asimismo, del total de la muestra considerada sólo el 24.3\% (equivalente a 146 ciudadanos de 600), considera que el uso de Internet propicia una interacción con las autoridades municipales que permita encontrar respuesta a las demandas de su comunidad. De esta forma, si bien es cierto que el uso de Internet propicia la interacción, eso no significa que mediante esta opción pueda resolverse de manera efectiva alguna problemática.

Por otra parte, se pensó necesario conocer desde la perspectiva ciudadana cuál consideran que es el uso más importante del portal web del gobierno municipal, y 
mayoritariamente, la respuesta fue simplificar trámites como puede verse en la gráfica 7, la participación ciudadana queda por debajo de cualquier otra opción de respuesta.

Gráfica 7. Uso más importante del portal gubernamental

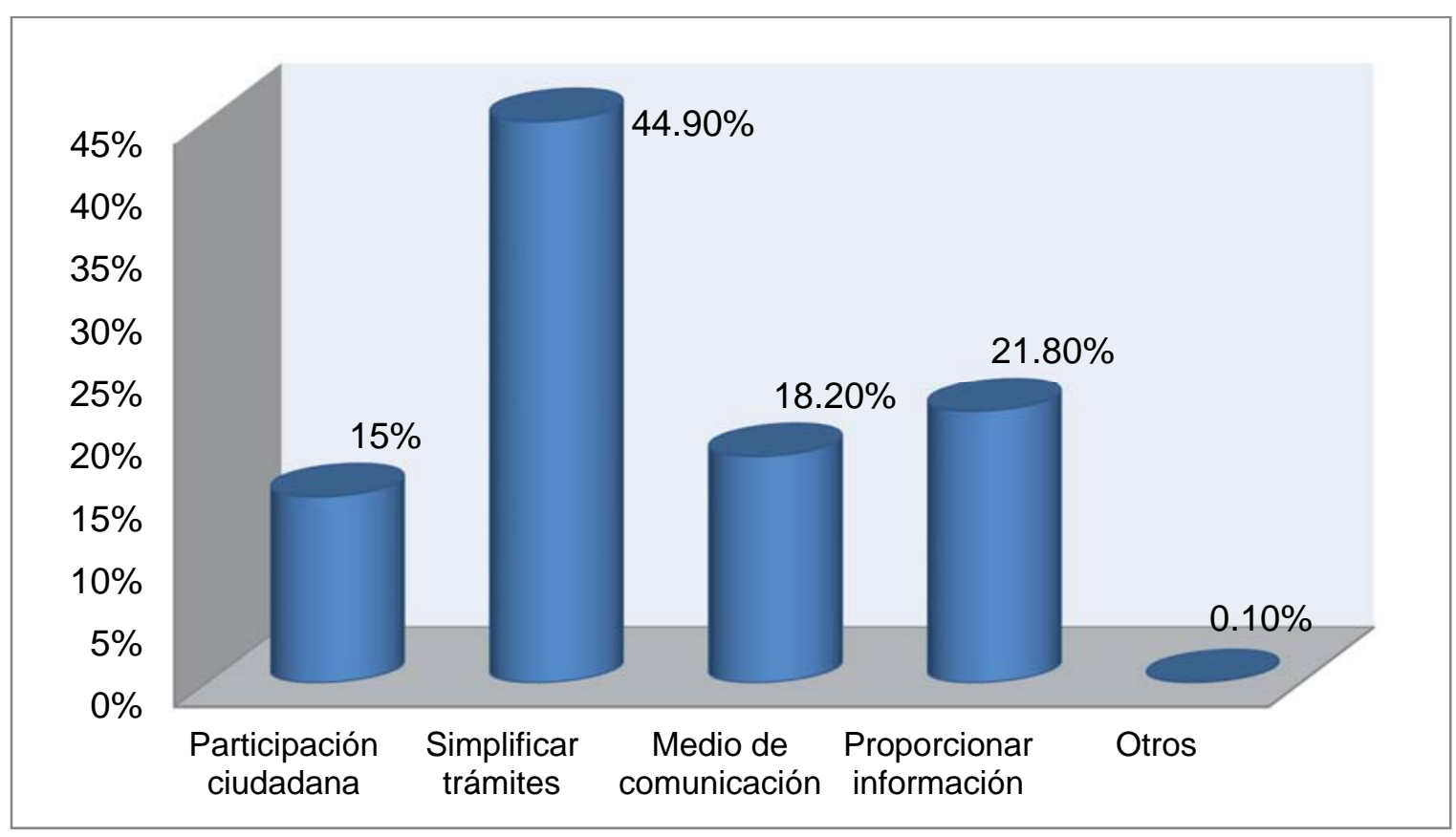

Fuente: Elaboración propia.

En general, la calificación otorgada por los ciudadanos al portal electrónico oscila entre 7 y 8 puntos, lo cual motiva mayor profundidad en el estudio.

\section{Discusión de los resultados y conclusiones}

Las condiciones del municipio de Toluca como capital de una de las entidades federativas más importantes del país, hacen suponer que el gobierno electrónico puede significar un elemento cotidiano en la relación gobierno- ciudadanos, sin embargo, con base en los resultados obtenidos se advierte que quienes hacen uso del portal electrónico o al menos 
están enterados de que existe, son mayoritariamente los ciudadanos con preparación educativa a nivel licenciatura, que se desarrollan en diversos empleos incluido el sector gubernamental y que tienen acceso a Internet en casa, en el ámbito laboral y a través de dispositivos móviles, lo cual facilita acceder al portal web.

Lo anterior abona a la reflexión sobre: hacia qué sector de la población se encaminan los esfuerzos de gobierno electrónico en el municipio. La utilización de las TIC permitiría a los gobiernos responder de manera más eficaz a las expectativas de los ciudadanos, pero los resultados obtenidos sugieren que un considerable número de ciudadanos no hacen uso del portal web, lo cual permite cuestionar acerca de la mejora en la prestación de servicios.

Sin embargo, como se señaló anteriormente, se puso especial atención en los elementos de la dimensión externa del gobierno electrónico señalados por Contreras (2014), oferta de información, prestación de servicios y participación democrática, por ello y con el fin de responder a la pregunta guía de esta investigación, se advierte que los ciudadanos utilizan el portal web principalmente para buscar información sobre las actividades del gobierno municipal o bien acerca del municipio, en segundo lugar la opción de realizar trámites o pagos de servicios y como última opción la participación o interacción.

Si la información que proporciona el portal web es el uso más destacado, significa que aún hay camino por recorrer, que el gobierno municipal debe contar con una estrategia para hacer del portal electrónico una herramienta que satisfaga de manera efectiva, eficiente y transparente los requerimientos ciudadanos.

Con base en el marco conceptual sobre gobierno electrónico, la oferta de información es necesaria, sin embargo, la parte más importante es la que se vincula a la mejora en la gestión y al logro de una administración eficiente. Con base en la calificación que los ciudadanos emitieron sobre la oferta de información y la prestación de servicios, se observa que fue de 8 puntos y para el rubro de participación /interacción fue de 7 puntos, en una escala del 1 al 10, donde 10 es la mejor calificación, por lo que se infiere que no hay una completa satisfacción de los usuarios cuando hacen uso del portal web. 
Se observa que la participación/ interacción a través del portal electrónico es el rubro que requiere mayor atención, ya que, de los 600 ciudadanos encuestados sólo 132 saben que existen canales para interactuar con su gobierno municipal y de esos ciudadanos, sólo 99 saben cuáles son esos medios de interacción. Esto refleja que, a pesar de los avances tecnológicos, la idea de gobierno electrónico no ha permeado tan profundamente como se podría esperar considerando el concepto más amplio.

La opinión de los ciudadanos, es un factor de relevancia que permite poner atención en el quehacer gubernamental e impulsar el establecimiento de una relación de confianza y colaboración, elementos que se aprecian ausentes con base en los resultados obtenidos.

Adicionalmente, si la percepción ciudadana se identifica con el sentir y la opinión de los ciudadanos, es necesario señalar que ellos reconocen que el uso más importante del portal web es la simplificación de trámites, aunque también es necesario subrayar la desconfianza que muestran (algunos ciudadanos) para realizar trámites en línea.

Este estudio y sus resultados son relevantes porque el gobierno electrónico debe ir dirigido a los ciudadanos y se aspira a que en esta época, determinada en muchos sentidos por los avances tecnológicos, la prestación de servicios pueda ser mejor, la gestión más eficiente, pero sobre todo que los ciudadanos puedan ser partícipes de la toma de decisiones o al menos de la solución de sus demandas, sin embargo, el uso de un portal electrónico todavía no contribuye del todo a un gobierno de proximidad.

Para pensar en la posibilidad de contar con un gobierno electrónico, es imprescindible considerar al ciudadano y sus necesidades. El avance tecnológico advierte que cada día las actividades de los ciudadanos se vinculan cada vez más al uso de las TIC, a pesar de que aún un gran porcentaje de la población parece ajeno los trámites electrónicos. Los gobiernos municipales deben marcar su propia línea de desarrollo en materia de gobierno electrónico, dadas las condiciones particulares de su contexto, no obstante, no pueden estar ajenos a las ventajas que representa centrar la atención en la prestación de servicios en el ciudadano.

Es necesario señalar que este estudio tiene limitaciones pues se trata solo de un municipio, y no es posible establecer generalizaciones, pero sienta las bases para 
continuar una investigación más profunda sobre el tema desde la perspectiva ciudadana, en donde mediante el diseño de otros indicadores se conozca la opinión sobre el desempeño gubernamental, la calidad en los servicios, el nivel de transparencia y rendición de cuentas de los gobiernos.

\section{Fuentes de información}

Alfaro, Rodrigo; Bustos, Guillermo; González, Alejandra y Loroño, Joseba (2005), Introducción al Gobierno Electrónico: Actores y Dimensiones, Chile: Ediciones Universitarias de Valparaíso, disponible en: http://www.euv.cl/archivos_pdf/gobiernoelectronico.pdf (fecha de consulta: 24 de agosto del 2017)

Contreras, Leticia (2014), "El cambio institucional y el gobierno electrónico", en Ruiz, Leobardo, Morales, Juan Miguel y Contreras, Leticia. (Coords.), Perspectivas del gobierno electrónico local en México, México: Universidad Autónoma del Estado de México, Instituto de Administración Pública del Estado de México, IAPAS.

Criado, Ignacio y Gil-García, José Ramón (2013), “Gobierno electrónico, gestión y políticas públicas: Estado actual y tendencias futuras en América Latina", en Gestión y Política Pública, Volumen temático, México: Centro de Investigación y Docencia Económicas.

Esteves, J. (2005), Análisis del desarrollo del gobierno electrónico municipal en España, Disponible en:

https://www.researchgate.net/profile/Jose_Esteves/publication/5013654_AnAlisis_del_des arrollo_del_gobierno_electrAnico_municipal_en_EspaAa/links/02e7e521da81bdf8770000 00/AnAlisis-del-desarrollo-del-gobierno-electrAnico-municipal-en-EspaAa.pdf (fecha de consulta: 6 de diciembre de 2017).

Estrada, S. (2007), Indicadores de gobierno electrónico: métricas derivadas de la experiencia de México. Red Iberoamericana de Indicadores de Ciencia y Tecnología, Disponible en: http://www.ricyt.org/manuales/doc_view/92-indicadores-de-gobierno- 
electronico-metricas-derivadas-de-la-experiencia-de-mexico (fecha de consulta: 4 de diciembre de 2017).

Fountain, Jane (2013), La construcción del Estado Virtual. Tecnologías de información y cambio institucional, México: Centro de Investigación y Docencia Económicas.

Gil-Garcia, José Ramón (2012), Enacting Electronic Government Success. An Integrative Study of Government-wide Websites, Organizational Capabilities, and Institutions, U.S.A.: Springer.

Instituto Electoral del Estado de México (2016), Estadístico de Padrón Electoral y Lista Nominal de Electores, corte al 31 de agosto de 2016, disponible en: http://www3.ieem.org.mx/numeralia/lista_nominal.html (fecha de consulta: 14 de enero del 2017)

INEGI (2010), Censo de Población y Vivienda 2010 disponible en: http://www.beta.inegi.org.mx/proyectos/ccpv/2010/_(fecha de consulta: 20de enero del 2017)

Mossberger, Karen; Wu, Yonghong y Crawford, Jared (2013), "Connecting citizens and local governments? Social media and interactivity in major US cities", en Government Information Quarterly, número 4, volumen 30.

Naser, Alejandra y Concha, Gastón (2011), El gobierno electrónico en la gestión pública, disponible en: http://www.cepal.org/es/publicaciones/7330-gobierno-electronico-la-gestionpublica_(fecha de consulta: 9 de febrero del 2017)

Pando, Diego (2010), Tecnologías de Información y Gestión del Cambio en las Administraciones Públicas, presentado en $\mathrm{V}$ Congreso Latinoamericano de Ciencia Política. Asociación Latinoamericana de Ciencia Política, Buenos Aires, disponible en: https://www.aacademica.org/000-036/172 (fecha de consulta: 2 de octubre del 2016)

Pando, Diego (2013), "Notas para una mayor y mejor utilización de las tecnologías de información en la gestión pública local" en Pando, Diego y Fernández, Nicolás (comp.), El gobierno electrónico a nivel local. Experiencias, tendencias y reflexiones, Buenos Aires, Argentina: CIPPEC. 
Pando, Diego y Fernández, Nicolás (comp.) (2013), El gobierno electrónico a nivel local. Experiencias, tendencias y reflexiones, Buenos Aires, Argentina: CIPPEC.

Ramírez, Álvaro (2010), “Innovación en la Gestión Pública y Open Government (Gobierno abierto): Una vieja nueva idea...", en Revista Buen Gobierno, número 9, disponible en: https://papers.ssrn.com/sol3/papers.cfm?abstract_id=1820326 (fecha de consulta: 7 de marzo del 2017)

Ramió, Carles y Miquel Salvador (2005), Instituciones y Nueva Gestión Pública en América Latina, Barcelona, España: Fundación CIDOB.

Sandoval, Rodrigo y Gil-García, José Ramón (2009), Propuesta de evaluación para portales de gobierno electrónico basada en el enfoque teórico evolutivo. Revista Chilena de Administración Pública, número 14, disponible en: http://revistas.uchile.cl/files/journals/48/articles/13504/public/13504-34813-1-PB.pdf (fecha de consulta: 8 de febrero del 2017)

Scott, James (2006), "E” the People: Do U.S. Municipal Government Web Sites Support Public Involvement?, en Public Administration Review, número 3, volumen 66, mayojunio, disponible en: http://onlinelibrary.wiley.com/doi/10.1111/j.15406210.2006.00593.x/full (fecha de consulta: 5 de abril del 2017)

Villoria, Manuel y Ramírez, Álvaro (2013), "Los modelos de gobierno electrónico y sus fases de desarrollo. Un análisis desde la teoría política", Revista Gestión y Política Pública, Volumen temático, México: Centro de Investigación y Docencia Económicas. 
El gobierno electrónico municipal en Toluca, Estado de México, desde la perspectiva ciudadana.

Leticia Contreras Orozco

$2, \frac{1}{4}$

E N C R U C I J A D A

REVISTA ELECTRÓNICA DEL

CENTRO DE ESTUDIOS EN

ADMINISTRACIÓN PÚBLICA

$28^{\circ}$ NÚMERO ENERO-ABRIL 2018

Revista Electrónica del Centro de Estudios en

Administración Pública de la Facultad de Ciencias

Políticas y Sociales, Universidad Nacional

Autónoma de México

UNIVERSIDAD NACIONAL AUTÓNOMA DE MÉXICO

Rector: Dr. Enrique Luis Graue Wiechers

Secretario General: Dr. Leonardo Lomelí Vanegas

Secretario Administrativo: Ing. Leopoldo Silva Gutiérrez

Abogada General: Dra. Mónica González Contró

\section{FACULTAD DE CIENCIAS POLÍTICAS Y SOCIALES}

Directora: Dra. María Angélica Cuéllar Vázquez

Secretario General: Mtro. Arturo Chávez López

Secretario Administrativo: Mtro. José A. Santiago Jiménez

Jefe de la División de Estudios de Posgrado:

Dr. Roberto Peña Guerrero.

Jefa de la División de Educación Continua y Vinculación: Mtra. Alma Iglesias González

Jefa de la División del Sistema Universidad Abierta y Educación a Distancia: Lic. Yazmín Gómez Montiel

Jefe de la División de Estudios Profesionales:

Dra. Martha Singer Sochet

Coordinador del Centro de Estudios en Administración Pública:

Dr. Maximiliano García Guzmán

Coordinador de Informática:

Ing. Alberto Axcaná de la Mora Pliego

w w w. p o I i i c a s. u n a m. m x
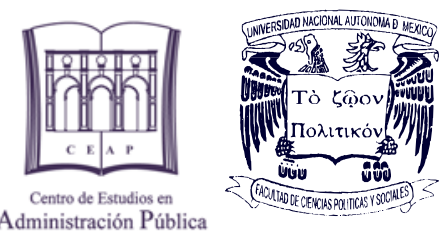

Administración Públiç FCPYS UNAM

\section{LA REVISTA}

Director de la Revista:

Dr. Maximiliano García Guzmán

Secretario Técnico de la Revista: Mtro. César C. Dionicio

Consejo Editorial:

Dr. Alejandro Navarro Arredondo

Dr. Arturo Hernández Magallón

Dr. Carlos Juan Núñez Rodríguez

Dra. Fiorella Mancini

Dr(c). Eduardo Villarreal

Dr. Roberto Moreno Espinosa

Diseño, integración y publicación electrónica: Coordinación de Informática, Centro de Investigación e Información Digital, FCPyS-UNAM.

Coordinación de producción: Alberto A. De la Mora Pliego. Diseño e Integración Web: Rodolfo Gerardo Ortiz Morales. Programación y plataforma Web: Guillermo Rosales García.

ENCRUCIJADA REVISTA ELECTRÓNICA DEL CENTRO DE ESTUDIOS EN ADMINISTRACIÓN PÚBLICA, Año 9, No.28, enero-abril 2018, es una publicación cuatrimestral editada por la Universidad Nacional Autónoma de México a través de la Facultad de Ciencias Políticas y Sociales y el Centro de Estudios en Administración Pública, Circuito Mario de la Cueva s/n, Ciudad Universitaria, Col. Copilco, Del. Coyoacán, C.P. 04510, México, D.F.,

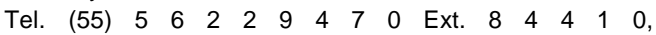
http://ciid.politicas.unam.mx/encrucijadaCEAP/, ceap@politicas.unam.mx. Editor responsable: Dr. Maximiliano García Guzmán. Reserva de Derechos al uso Exclusivo No. 04-2011-011413340100-203, ISSN: 2007-1949. Responsable de la última actualización de este número, Centro de Estudios en Administración Pública de la Facultad de Ciencias Políticas y Sociales, Mtro. César C. Dionicio, Circuito Mario de la Cueva $s / n$, Ciudad Universitaria, Col. Copilco, Del. Coyoacán, C.P. 04510, México D.F., fecha de la última modificación, 12 de enero de 2018.

Las opiniones expresadas por los autores no necesariamente reflejan la postura del editor de la publicación. Se autoriza la reproducción total o parcial de los textos aquí publicados siempre y cuando se cite la fuente completa y la dirección electrónica de la publicación 